\title{
Primary and Secondary Reference Materials for Procedures to Test the Quality of Medicines and Foods
}

\author{
USP Council of Experts • USP Reference Standards Committee • Walter W. Hauck
}

Received: 11 August 2011 / Accepted: 17 January 2012 / Published online: 8 February 2012

(C) Springer Science+Business Media, LLC 2012

\begin{abstract}
At present a complex global patchwork of private and public monographs and reference materials is variously available to help ensure the quality of medicines and foods. The relationship of these monographs and reference materials, one to another, frequently is inconsistently understood and documented. This article considers the complexity of monographs and reference materials with a focus on qualifying one reference material relative to another.
\end{abstract}

KEY WORDS comparability · metrology ' quality assurance . reference materials $\cdot$ standards

\section{INTRODUCTION}

The discovery, development, and registration (approval and licensing) process brings medicines to a market with defined safety and associated quality attributes. The quality attributes allow continuing market surveillance. Analogous efforts for foods, including foods, food ingredients, processed foods, and (in the United States) dietary supplements, allow the same opportunity. Associated with market access and surveillance is the availability of a private or public documentary (control) standard, termed a monograph, with one or more reference materials to assist in conformity testing for quality. Typically the monograph consists of primary

Members of USP Council of Experts and USP Reference Standards Committee are listed in Appendix I and II.

W. W. Hauck $(\triangle) \cdot$ USP Council of Experts ·

USP Reference Standards Committee

US Pharmacopeial Convention

1260| Twinbrook Parkway

Rockville, Maryland 20852- 1790, USA

e-mail:wh@usp.org information (e.g., definitions and labeling and storage statements) followed by the specification for the article - its tests, procedures, and acceptance criteria that should be met throughout its life cycle. Monographs and their allied reference materials may be used by first parties (manufacturers and compounding professionals), second parties (purchasers), and/or third parties (governmental bodies and others independent of the supplier and purchaser) as a product standard to allow testing. The availability of these product testing standards allies with staff education and training (people standards), process standards such as good manufacturing practices (CGMP), and good supply chain management and good storage and distribution practices.

If the entire system, from development through registration and market surveillance, works properly it instills confidence in manufactured medicines and foods through a series of wellmaintained safety nets. If the system does not work properly, massive and systematic failures can occur, e.g., catastrophes associated with diethylene glycol, melamine, and contaminated heparin. These might be regarded as the tip of the iceberg because of unknown or unreported instances when adulterated medicines and foods are used by patients and consumers as provided by both ethical and fraudulent manufacturers. Indeed, the entire regulatory and compendial posture of many countries, including the United States, is designed to detect and deter adulterated medicines and foods (1). A particular problem is the absence of publicly available reference materials. This material is not merely nice to have but is critical. Without the availability of this public material, no second or third party can test independently or take action. This availability is thus fundamental to the need to assure all partiesfirst, second, and third - that medicines and foods are manufactured according to up-to-date and relevant standards. Reference materials are an integral component of the procedures of the private or public control specification. The US Pharmacopeial Convention (USP) works with the US Food and 
Drug Administration (FDA) and other regulatory agencies throughout the world, as well as their official medicines control laboratories, to provide publicly available reference materials for the United States Pharmacopeia (USP) and National Formulary $(\mathcal{N} F)$, the USP Dietary Supplement Compendium, the USP Food Chemicals Codex, the Pharmacists' Pharmacopeia, and USP's new Medicines Compendium. Such cooperation is equally true of other pharmacopoeias (for medicines) and compendia and codexes (for foods) throughout the world.

USP's monograph and reference material standards may be or have been taken up in law, regulation, and guidance in the United States and other countries. At times via formal and informal agreements, USP allows adoption or adaption, without charge, of monograph text into compendial text of another country. A manufacturer also may use a USP standard in a private regulatory filing as a means of assuring regulators that its products and their ingredients meet acceptable standards for purposes of market access and surveillance (2). These statements apply generally to other pharmacopeial and compendial/codex texts of the world, and many official and nonofficial producers of these textsbut not all—provide publicly available reference materials that support the procedures of the monographs. Regulatory agencies also may provide publicly available monographs and reference materials for articles of medicines and food commerce. At times, however, public monographs and reference materials are not readily available for many years after market access, if at all, from any regulatory agency, from USP or from any other pharmacopoeia.

Although USP reference materials must be used to conclusively demonstrate compliance with USP compendial standards, ${ }^{1}$ many manufacturers and other parties may use alternative procedures and secondary reference materials in the course of testing to maintain requisite quality. They may do this for a number of reasons, including cost, convenience, assured supply, or absence of publicly available material. In the absence of publicly available reference materials, manufacturers often establish in-house primary reference materials. This paper addresses the need for qualification comparisons to help ensure that in-house reference materials are properly compared against official or authorized materials so that the possibility of introducing differences is

\footnotetext{
${ }^{1}$ For purposes of legal compliance with regulations in the United States, an article is conclusively shown to meet a USP compendial standard only when tested as directed in the relevant monograph and applicable general chapters using USP reference materials that have been approved as suitable for use as comparison standards in USP or $\mathcal{N F}$ tests and assays (3). Under federal law in the United States, to avoid being deemed adulterated or misbranded, drugs (and dietary supplements labeled USP) must comply with USP compendial standards. The topic of the relationship between the tests, procedures, and acceptance criteria of a monograph has been considered elsewhere (4-6).
}

minimized. A further and continuing issue is whether a reference material is officially recognized in the absence of a publicly available regulatory or compendial standard. An even deeper and more complex issue arises whenever a regulatory or compendial body either is lacking or is so inadequately resourced that patients, practitioners, and consumers can rely only on first parties (manufacturers) for assurance of the quality of their medicines and foods. This need not be a problem with highly committed and ethical manufacturers, but it disallows independent checks, which are a fundamental presumption of buyer-seller transactions.

The relationship of standards and materials frequently is poorly understood and rarely documented publicly. For clarification, this paper presents and discusses the following five examples.

Example 1 Transition from the first-entry manufacturer's reference material arising from commitments made to national regulatory authorities (termed a house primary standard) to the national primary reference material prepared by the pharmacopeia

Example 2 Preparation of a secondary reference material by comparison to the national primary reference material (or a manufacturer's preparing a secondary reference material and relating the latter to its house primary standard when a national primary reference material is not available)

Example 3 Establishment of the suitability for use of a national primary reference material by a pharmacopeia that has adopted or adapted compendial text from another pharmacopeia in order to qualify the available reference material to the applicable adopted/adapted procedure

Example 4 Qualification of one national primary reference material by comparison to another

Example 5 Qualification of a national primary reference material to support a monograph procedure in another pharmacopeia

Other permutations are possible, but these examples help illustrate principles and approaches that should be considered when one reference material is qualified to another and both relate to one or more compendial procedures. This article focuses on chemical reference materials for medicines, although many of the concepts considered are similar to those for biological reference materials for medicines and foods. We note that standardization of food additive/ ingredient monographs sometimes proceeds more strongly at a global level via Joint FAO/WHO Expert Committee on Food Additives (JECFA) monographs than for medicinal ingredients. Further we speak in the article to national requirements and recommendations with the understanding that global and/or regional ones also may exist. 
Following this introduction we provide three sections: The first considers some basic metrological considerations related to reference materials. This provides a necessary background and terminology for the remainder of the article. As will be seen in the second section, the terminology in this area is not consistent across agencies and organizations. Accordingly in the second section we present key definitions and concepts related to the use of primary and secondary reference materials. The third section presents uncertainty considerations related to reference materials. We conclude with a discussion section that revisits the five cited examples.

\section{NOMENCLATURE}

The definitions in this section follow the International Vocabulary of Metrology (VIM) (7). Definitions from other sources are given in the following section, "Definitions".

VIM defines reference material as "material, sufficiently homogeneous and stable with reference to specified properties, which has been established to be fit for its intended use in measurement or in examination of nominal properties" (7). This paper uses the term qualified to mean established to be fit for its intended use. VIM also defines reference standards (see "Definitions"), and there is little difference between VIM's definitions for reference materials and standards. Use of materials rather than standards is the current trend and will be used in this article. Such usage makes clear that the standards of primary interest are, in fact, materials and not some other type of documentary (normative) standard. Reference materials are certified (certified reference materials, CRMs) if they are "accompanied by documentation issued by an authoritative body and providing one or more specified property values with associated uncertainties and traceabilities, using valid procedures" (7).

Uncertainty according to VIM is a "non-negative parameter characterizing the dispersion of the quantity values being attributed to a measurand [quantity intended to be measured], based on the information used. Uncertainty is usually expressed as a standard deviation" (7). Uncertainties can be Type $\mathrm{A}$ or Type $\mathrm{B}$, depending on whether these are obtained by statistical means (A) or other means (B), e.g., from a certificate from an equipment manufacturer.

Metrologically comparable measurement results are "metrologically traceable to the same reference," and metrological traceability is the property of a measurement result whereby the result can be related to a reference by a documented unbroken chain of measurements, each contributing to the measurement uncertainty (7). Metrological traceability to a common reference material [preferably associated with Système International (SI) units] ensures consistency and comparability of measurements across time, space, and technology (6). Such consistency and comparability ensure that, for example, the $\mathrm{mg}$ (or unit, generally) is the same for all products sold. Ensuring traceability means that the content of secondary materials is determined relative to that of a higher-order material, whether the latter is national (e.g., USP) or international [e.g., the World Health Organization (WHO)]. In addition, as noted by the International Organization for Standardization (ISO), "Any traceability [of a CRM] defined in principle only, but without an evaluation of the uncertainty, does not constitute a properly demonstrated traceability" (8).

The traceability hierarchy is shown in Fig. 1 for the case in which $U S P-N F$ is the applicable national standard in the US and in other countries that recognize USP standards either in law or regulatory filings. This figure also makes clear that each procedure on this ladder is associated with a reference material and that as consecutive measurements are undertaken farther down the ladder, the relative uncertainty associated with the result must increase.

\section{DEFINITIONS}

Following are definitions and requirements or recommendations from metrology and food and drug regulators regarding reference materials.

\section{VIM}

As noted, VIM defines both reference materials and reference standards (7). Reference standards, or reference measurement standards, are "measurement standards designated for the calibration of other measurement standards for quantities of a given kind in a given organization or at a given location" (7). A measurement standard is a "realization of the definition of a given quantity, with stated quantity value and associated measurement uncertainty, used as a reference" (7). Measurement standards can be primary or secondary, and secondary standards are "established through calibration with respect to a primary measurement standard for a quantity of the same kind."

\section{ISO}

ISO currently defines reference material differently than VIM but comes into close agreement with a recent revision to its Guide 30 (9). The ISO revision defines reference material as a "material, sufficiently homogeneous and stable with respect to one or more specified properties, which has been established to be fit for its intended use in a measurement process" and notes that properties can be quantitative or qualitative.

ISO Guide 30 defines a primary standard as one "that is designated or widely acknowledged as having the highest metrological qualities and whose value is accepted without 


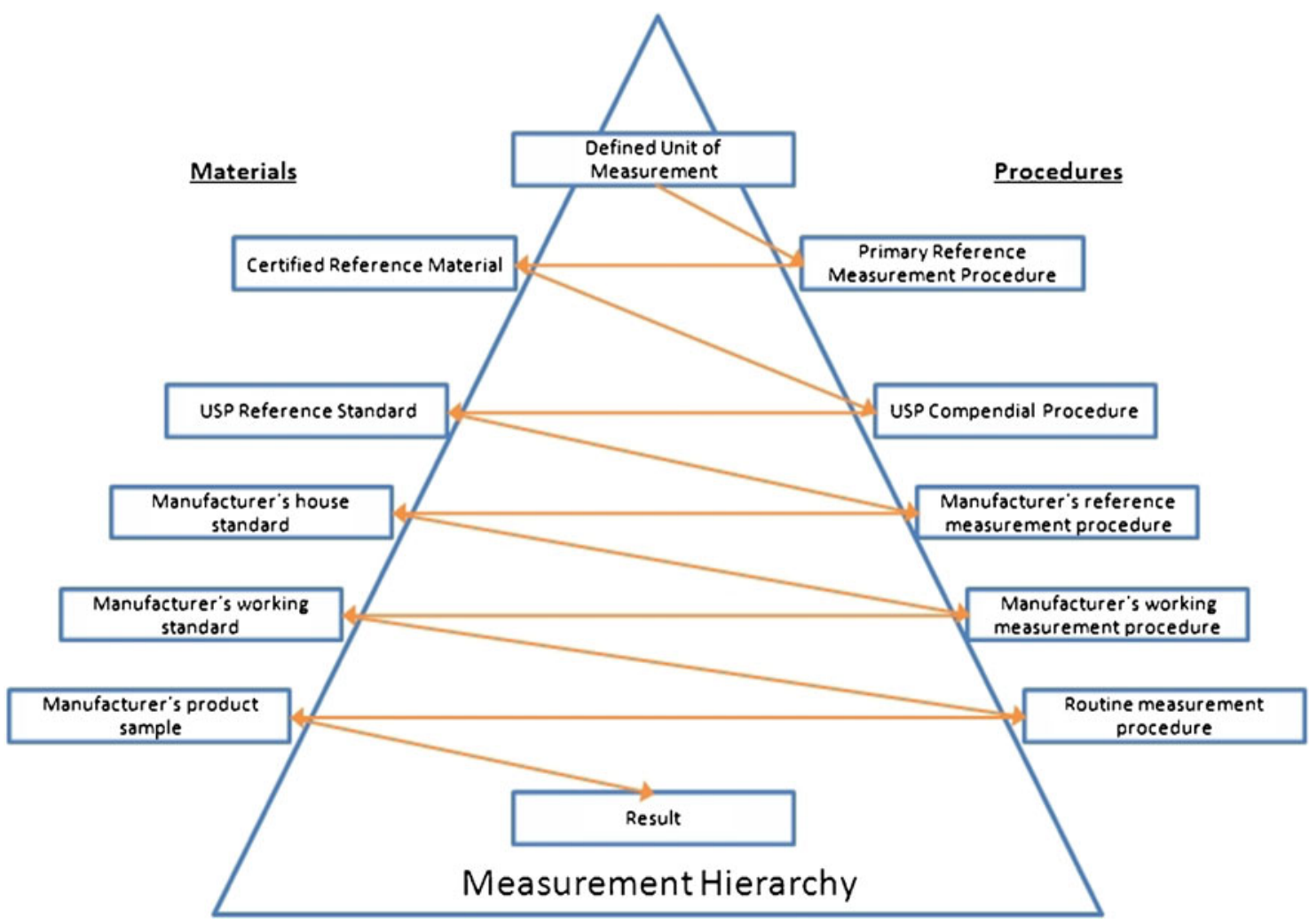

Fig. I The measurement hierarchy applied to pharmaceuticals, the ideal state. This figure traces a result back to a defined unit of measurement as links with a hierarchy of materials and procedures. Uncertainty in results increases as one goes down the path.

reference to other standards of the same quantity, within a specified context" and a secondary standard as one "whose value is assigned by comparison with a primary standard of the same quantity" (9). ISO Guide 33 "strongly recommend[s] that CRMs always be used instead of in-house standards because of the resultant enhanced confidence in the measurement output" (10). The only caveat is for some uses and for CRMs that are in short supply or are very expensive. ISO Guide 33 adds that "it is important that users remain aware that the preparation of in-house standards for use instead of CRMs has an associated cost based on factors such as material cost, facility usage charges, personnel labor rates, etc., in which the material cost is in general the lowest" (10). For calibration laboratories, ISO International Standard 17025 requires that the "laboratory shall have a program and procedure for the calibration of its reference standards. Reference standards shall be calibrated by a body that can provide traceability ... Reference materials shall, where possible, be traceable to SI units of measurement, or to certified reference materials. Internal reference materials shall be checked as far as is technically and economically practicable" (11).

\section{WHO}

WHO uses the term primary chemical reference substance to mean "one that is widely acknowledged to have the appropriate qualities within a specified context and whose assigned content when used as an assay standard is accepted without requiring comparison to another chemical substance" (12). A secondary chemical reference substance is one "whose characteristics are assigned and/or calibrated by comparison with a primary chemical reference substance" (12). For biologics, WHO uses different terminology: "WHO biological reference standards comprise materials of complex composition that require biological or immunological assay for appropriate characterization" (13). Its current list of available biological reference materials uses biological reference preparation as the overarching term, and specific items are termed international standards, international reference preparations, international reference reagents, or reference reagents (14). WHO notes that "it is essential that a secondary reference substance is traceable to a primary reference substance, such as a pharmacopoeial or other officially recognized reference substance" (12). Although WHO directs this comment to regional/national standards, WHO adds that "in principle, secondary reference standards prepared by manufacturers can be prepared as 'working standards' using the same procedures" (12). WHO also notes some of the uses of reference materials for national pharmaceutical control laboratories: "Reference materials (e.g., official reference substances and reference preparations, secondary reference materials, and nonofficial materials prepared in the laboratory as working standards) are necessary for the testing and/or calibration, 
validation, or verification of a sample or of equipment, instruments, or other devices" (15).

\section{FDA and ICH}

FDA, in its guidance on Analytical Procedures and Methods Validation in the drug application (not pharmacopeial) context, states that "a reference standard (i.e., primary standard) may be obtained from the USP-NF or other official sources ... A working standard (i.e., in-house or secondary standard) is a standard that is qualified against and is used instead of the reference standard" (16). FDA adds that a working standard must be qualified against a primary reference standard (including the requirement that the reference standard be "thoroughly characterized to ensure its identity, strength, quality, purity, and potency"). This guidance includes a page and a half of requirements that must be satisfied if the company chooses to use its own reference material instead of one from an official source such as USP. It adds, "Reference standards from $U S P-\mathcal{N} F$ and other official sources do not require further characterization" (16).

Similar considerations appear in other FDA documents. An Office of Regulatory Affairs guidance discussing inspections of biotechnology manufacturing facilities also suggests that reference standards for potency can be qualified against one of several types of reference standards, where available: "Where applicable, in-house biological potency standards should [emphasis added] be cross-referenced against international [WHO, National Institute for Biological Standards and Control], or national [National Institutes of Health, National Cancer Institute, FDA] reference standard preparations, or USP standards" (17). Also, this guidance points out the following cGMP deficiencies that may warrant regulatory action if detected during an inspection of a drug substance manufacturer: "If there is a USP reference standard, failure to test each lot of an in-house (secondary reference standard) against the USP primary reference standard before use" (18).

A FDA internal guidance applicable to satisfying CGMP (not compendial) requirements states, "We generally recommend use of official reference standards for analysis of compendial articles. However, use of secondary reference standards is acceptable if each [secondary reference standard] lot's suitability is determined prior to use by comparison against the current official USP reference standard and each lot is requalified periodically in accordance with a written protocol. The protocol should clearly address the receipt, storage, handling and use of primary reference standards, the purification of secondary standards, and their qualification against USP reference standards [emphasis added]" (19).

The International Conference on Harmonization (ICH) uses similar language relating to satisfaction of CGMPs but not necessarily compendial standards in ICH Q7:
Where a primary reference standard is not available from an officially recognized source, an "in-house primary standard" should be established. Appropriate testing should be performed to establish fully the identity and purity of the primary reference standard. Appropriate documentation of this testing should be maintained ... Secondary reference standards should be appropriately prepared, identified, tested, approved, and stored. The suitability of each batch of secondary reference standard should be determined prior to first use by comparing against a primary reference standard. Each batch of secondary reference standard should be periodically requalified in accordance with a written protocol (20).

\section{Pharmacopeias}

$U S P-\mathcal{N} F$ states that "USP Reference Standards are authentic specimens that have been approved as suitable for use as comparison standards in USP or NF tests and assays" (21). They are further described as "highly characterized specimens reflective of specified drugs and foods (drug substances, biologics, excipients, dietary supplements, food ingredients, impurities, degradation products, reagents, and performance verification standards)" (21). The term reflective is key: USP's reference materials (and those of other pharmacopeias) are not medicines, but rather chemicals or biologics. They are not used for food or medicinal purposes, are not subject to applicable CGMPs, and are accordingly labeled.

In the European Pharmacopoeia, reference standard is used "as a general term covering reference substances, reference preparations, and reference spectra," and primary standard is "a standard shown to have suitable properties for the intended use, the demonstration of suitability being made without comparison to an existing standard" (22). A secondary standard is

a standard established by comparison with a primary standard ... A secondary standard may be used for routine quality control purposes for any of the uses described above for primary standards, provided that it is established with reference to the primary standard. A secondary standard is established and employed to reduce the use of the primary standard, which requires more extensive characterization and evaluation and may be available only in a limited quantity. A secondary standard is used only for the same purpose as the primary standard with reference to which it has been established (22).

A directive from the European Parliament specifies traceability as an essential requirement for in vitro diagnostic devices: "The traceability of values assigned to calibrators and/or control materials must be assured through available 
reference measurement procedures and/or available reference materials of a higher order" (23). This approach is lacking in many other countries and regions of the world, including the United States.

\section{Food Codexes and Compendia}

General principles applicable to medicines and their ingredients apply as well to foods. In the absence of strong regulatory control, national (or global) primary standards do not exist. However principles of metrology argue that either an independent body or a manufacturer may produce a primary reference material in accordance with VIM definitions. For example, the Codex Alimentarius calls for use of certified reference materials for evaluation of trueness as part of validation of procedures (24).

\section{UNCERTAINTY CONSIDERATIONS}

The value assigned to a reference material, either primary or secondary, has an uncertainty. This is true even if the uncertainty has not been determined or has been determined but is not stated on a label or certificate. In use, the error in the assigned value for a given lot of a reference material is constant across use. Reference material uncertainty manifests itself only when a laboratory transitions from one lot of the reference material to another and results appear to shift. If the error in assigning a value to a reference material were known in this transition, it would be treated as a bias, and analysts would correct accordingly. Without this understanding, no correction is possible. Because it is not known, it is treated as an uncertainty.

The value assigned to the secondary material has two components. The first is a ratio, often of areas or response factors or a relative potency comparing the secondary reference material to the primary reference material. Second, there is the multiplication of the ratio by the value assigned to the primary material in order to establish the value for the secondary reference material. Both steps contribute to the uncertainty of the value assigned to the secondary reference material, but only the magnitude of the first is within the control of the company developing the secondary material.

For $S=P^{*} R$, by propagation of errors, we have:

$u n c_{S}^{2} \approx P^{2} u n c_{R}^{2}+R^{2} u n c_{P}^{2}$

where $S, R$, and $P$ denote assigned value of the secondary reference material, ratio, and assigned value of the primary reference material, respectively, and unc denotes corresponding uncertainty carried by that parameter. A manufacturer can make the type $\mathrm{A}$ component of $u n c_{R}$ arbitrarily small by its choice of procedure(s), experimental design, and sample size. However, $u_{n} c_{P}$ is determined by the supplier of the primary reference material and is a fixed value for the maker of the secondary material. So, regardless of the amount of work done by the company, the uncertainty of the secondary reference material will include a component due to the primary reference material. Because the ratio, $R$, normally is near 1.0, the uncertainty of the value assigned to the secondary reference material typically will exceed that of the value assigned to the primary material.

Increasing the uncertainty of a reference material leads to a need for tighter acceptance criteria in specifications that make use of that reference material. The importance of this extra uncertainty depends on how important the uncertainty is relative to the remaining uncertainty of the procedure and product. For example, if the materials are highly pure small molecules and the dominant variability arises from product manufacturing, the increase in uncertainty of the secondary reference material often is inconsequential. Yet the larger issue of understanding manufacturing variability - a key component of the current impetus undergirding quality by design (25-28) - is fully aligned with and is consonant with the understanding of variability in test results embodied in determination of uncertainty of the analytical procedure and the reference materials used in the analytical procedure. The target for all these efforts is necessarily the private or public specification with tests that reflect the critical quality attributes, procedures for these tests, and acceptance criteria.

\section{DISCUSSION}

Nearly all procedures of a private or public control monograph require a reference material in order to meet standards of sound measurement science. Beyond this general statement are at times confusing sets of nomenclature, although the underlying metrologic principles and expectations generally are clear (see Fig. 1) (29). Nomenclature and metrologic approaches allow understanding that there is a chain in which a primary procedure and reference material can be associated with succeeding ones (30). A key point is whether these are publicly available. In most jurisdictions, the national primary procedure and reference material exist as part of a regulatory filing and subsequently may enter the public domain only many years after initial marketing. For purposes of this discussion, we use the term primary house reference material to denote a reference material that is not publicly available and for which no national, regional, or global reference material (or public monograph) exists. This material also may have subsidiary house reference materials that are not publicly available. When the national primary reference material becomes available, this primary house material 
becomes a secondary house material. These observations lead to conclusions relative to the examples provided at the beginning of this article and are summarized as follows:

Example 1 Transition from the first-entry manufacturer's reference material arising from commitments made to national regulatory authorities (termed a house primary standard) to the national primary reference material prepared by the pharmacopeia.

In this example, the public global/national primary reference material becomes the material "whose assigned content when used as an assay standard is accepted without requiring comparison to another chemical substance" (WHO definition; see above). The primary house reference material may be retired, requalified compared to this national primary material, or retained for other purposes (e.g., to serve as a primary house reference material for other regions of the world where a national primary material is not yet available). Most of these scenarios have the potential to introduce higher uncertainly in measurement results (see "Uncertainty Considerations," above).

These principles hold true even if the material of the primary house standard is "identical" to the reference material prepared and disseminated by the regulatory and/or pharmacopeial/compendial authority. A reference material per se has no particular meaning-only when coupled with independent characterization and collaborative testing does it become associated with useful information, particularly in the case of a CRM for which a certificate with required components amplifies the material's value. Furthermore, the public reference material necessarily is separated from its sources not only in terms of its sources but in terms of how it is maintained, prepared, and used.

Example 2 Preparation of a secondary reference material by comparison to the national primary reference material (or a manufacturer's preparing a secondary reference material and relating the latter to its house primary standard when a national primary reference material is not available).

In this example, a detailed protocol must be developed to direct qualification of the secondary material by reference to the primary material. The uncertainty of the secondary material will increase.

Example 3 Establishment of the suitability for use of a national primary reference material by a pharmacopeia that has adopted or adapted compendial text from another pharmacopeia in order to qualify the available reference material to the applicable adopted/adapted procedure.
In this example, a general protocol must be developed to allow suitable studies in which a candidate reference material from any source is allied appropriately with the adopted/adapted compendial text. This reference material will not require comparison to another chemical substance (WHO definition; see above). Uncertainty is established for the first time, and the material becomes the national or regional primary reference material.

Example 4 Qualification of one national primary reference material by comparison to another.

In this example, a general protocol must be developed for suitable studies in which a candidate reference material and procedures across compendia and from different reference material sources yield information for subsequent decisionmaking. The decision-making involves selection of a suitable primary material to which all other materials can be compared as secondary materials with increased uncertainty.

Example 5 Qualification of a national primary reference material to support a monograph procedure in another pharmacopeia.

In this example, a general protocol must be developed to allow suitable studies so that the national primary reference material is qualified appropriately relative to the procedures in the monograph of the other country and region. This material does not require comparison to another chemical substance (WHO definition; see above). Uncertainty does not increase.

Based on these examples, significant complexity and additional costs clearly arise as a result of the patchwork environment that now exists for reference materials relative to private or public monographs. Issues include public health costs, costs of qualifying secondary and subsidiary reference materials, and the costs associated with the increased uncertainty that arises with qualification of secondary and subsidiary materials. In particular, the existence of multiple national primary reference materials raises several concerns: 1) it introduces the potential for bias in drug dosing between regions (a public health issue); 2) it is a duplication of effort for regulatory and compendial agencies (increases costs for citizens); 3) and it imposes a burden on global manufacturers to show traceability to more than one primary reference material (increases complexity that can affect public health and increases the cost of health care).

When a public reference material becomes available following regulatory or compendial action, a transition from a primary house reference material to a national primary reference material occurs. USP's approaches and experience are paradigmatic for this transition. USP typically relies on a manufacturer's donation of the primary house reference material (typically the medicinal ingredient itself and one or more impurities), or USP obtains this candidate 
material by purchase or synthesis. USP then qualifies the candidate material by intensive independent studies that include collaborative testing to yield a value assignment, i.e., a stated content of the molecule or mixture of interest relative to the sum of the molecule/mixture and its impurities. USP can add an uncertainty statement to this value assignment that, together with additional information and a certification process, may allow it to become a CRM. The uncertainty statement is valuable to users who wish a more comprehensive understanding of the sources of variability and bias that can influence their ability to meet test specifications.

When new USP reference materials are established, all data from these activities are submitted to USP's Council of Experts for review and decision-making. The result, if the Council of Experts agrees, is a national primary reference material for use in the United States and in any other jurisdiction where the USP reference material is recognized as suitable for one or more procedures of a monograph (7). As the USP public reference material becomes available, the purpose of the manufacturer's house reference material must be reconsidered, and any secondary material must be shown to be equivalent to the new USP reference material. USP maintains an intensive continued suitability-for-use program to ensure stability over time in lots of its reference materials. USP does not make secondary standards. Approaches in other jurisdictions, both for medicines and foods, generally follow those that exist for USP_-but there are many variations, gaps, additions, and deletions.

In the United States and other jurisdictions, the compendial monograph and material, if they are available, are used for purposes of regulatory compliance. A first party is responsible for avoiding improper use of this uncertainty statement, e.g., in the disposition of individual test results, and the reference material is subject to further conformity assessments. Thus, the uncertainty statement is valuable to first, second, and third parties who require a full understanding of the sources of variability and bias that can influence their ability to meet test specifications.

The information provided from various regulatory bodies (see "Definitions") in this report emphasizes two key points: First, manufacturers find a safe harbor in choosing a reference material from a recognized official or authoritative source. Second, the studies needed to ensure that a secondary material is qualified to substitute for a primary reference material are not trivial. When further lots of a secondary material are needed or when the national primary batch changes, then requalification to the primary material is required. Data must be available to demonstrate that assigned values of secondary materials will generate equivalent results when compared to higher-order materials, i.e., that results using different secondary materials will be metrologically comparable.
Most industries follow ISO, which means that values assigned to secondary reference materials must be traceable to higher-order reference materials. WHO calls for traceability as well for national and regional reference materials. The pharmaceutical industry follows FDA and ICH language that the secondary material is qualified by comparison to a primary material, but guidances do not clarify the meaning of comparison to. Our understanding from industry colleagues is that comparison often means that results using a primary reference material are compared to results using the secondary material with the goal of demonstrating sufficiently similar results without necessarily including uncertainty considerations in the comparison. This is an indirect approach to comparison of the reference materials, with value assignment to the secondary material done without comparison to the primary material (e.g., by mass balance).

Studies are needed to ensure comparability of results obtained from national primary reference material and results from secondary or subsidiary reference material. These include characterization or recharacterization studies as well as collaborative testing. Variability in results of this testing arises from laboratories, procedures, and reference materials (31). The design and execution of the studies needed to characterize and to assign values require careful consideration so that reasonable conclusions can be drawn from results. Examples are available in pilot studies and key comparisons of the Bureau International des Poids et Mesures (BIPM) (see http://kcdb.bipm.org) as well as in the collaborative studies conducted by WHO for biological reference materials.

A claim that a primary house or secondary reference material is traceable to a USP or other higher-order reference material relies on well-designed and wellexecuted studies. If a reference material manufacturer claims traceability of the content of its material to USP's reference material, then the content of the secondary material has been assigned by comparison to the USP material (or to a higher-order material to which the content of the USP material is traceable, such as a WHO reference material), and the comparison must be maintained over time. The latter requires periodic recomparison of test results using the different reference materials.

As shown in the "Uncertainty Considerations" section above, uncertainly for a secondary or subsidiary reference material necessarily is greater than that of the primary reference material to which it is compared. In many instances this increase in uncertainty may be trivial, but this understanding is based on the uncertainty of the primary material itself. Extremely pure reference materials may lead to a relatively unimportant increase in uncertainty, but less pure reference materials (such as for biologics and impurities) may lead to increases that are problematic (31). 


\section{CONCLUSIONS}

This report summarizes relationships between private and public primary and secondary reference materials for the procedures in controlling monographs for foods and drugs. Although these relationships are complex and the nomenclature varies, the overarching scientific components are increasingly clear. For medicines and foods, a national or regional or even global goal might be a comprehensive collection of public documentary and reference material standards that can be used by first, second, and third parties to test for quality. This would support concepts embodied in US law and elsewhere that documentary and reference material standards are used in an official compendium. An even more ambitious goal might be a single collection of documentary standards and reference materials to support testing of medicines and foods. If achieved, this goal would support the metrological objective of one test that is accepted everywhere without regard to time or space (i.e., "once tested, everywhere accepted"). National standards would be qualified and would be traceable to this more general standard. This harmonizing goal has proceeded at a relatively slow place both for medicines and their ingredients and for foods and for processed foods and their ingredients. Yet the scientific understanding of the overall challenge may allow a more rapid achievement of what once appeared to be a remote possibility. With availability of monographs where multiple acceptable procedures might be allowed (and one reference procedure designated for purposes of compliance) $(6,32)$, coupled with certified and other reference materials as needed, it is possible to imagine that a full cohort of public procedures and reference materials may become available in the coming years to help ensure the quality and benefit of medicines and foods. The approach relies on and is amplified by the availability of CRMs. Many specific agreements and solutions must be worked out among regulatory agencies, pharmacopeial bodies, and medicines and foods manufacturers to advance to this opportunity, but the overall benefit to all and especially to patients and consumers is increasingly clear.

\section{ACKNOWLEDGMENTS \& DISCLOSURES}

The authors thank Stefan Schuber, PhD, ELS, of USP for editorial assistance and Matthew Van Hook, JD, of USP for advice about legal considerations.

\section{APPENDIX I: MEMBERS OF THE USP COUNCIL OF EXPERTS}

Roger L. Williams, MD (Chair); James E. Akers, PhD; Gregory E. Amidon, PhD; Lawrence H. Block, PhD;
Matthew W. Borer, PhD; Michael A. Cutrera, MSc; Gigi S. Davidson, BSPharm, DICVP; James E. De Muth, PhD; Andrew G. Ebert, PhD; Mary G. Foster, PharmD, BFA; Antony Raj Gomas, PhD; Dennis K.J. Gorecki, BSP, PhD; Jean F. Huxsoll, PhD; Michael G. Mulkerrin, PhD; Bernard A. Olsen, PhD; Robert E. Osterberg, PhD; Ernest Parente, PhD; Thomas P. Reinders, PharmD; Robert R. Singer, MSc; Glenn A. Van Buskirk, PhD; Wesley E. Workman, PhD; Timothy J. Wozniak, PhD

\section{APPENDIX 2: MEMBERS OF THE USP REFERENCE STANDARDS COMMITTEE}

Matthew W. Borer, PhD (Chair); Antony Raj Gomas, PhD (Vice-Chair); Bianca Avramovitch, PhD; Adrian F. Bristow, $\mathrm{PhD}$; Shaohong Jin; Catherine A. Rimmer, PhD; Iffaaz M. Salahudeen, PhD; Ralph E. Sturgeon, PhD; Robert L. Watters, PhD; M.L. Jane Weitzel

\section{REFERENCES}

1. Dudzinski DM. Reflections on historical, scientific, and legal issues relevant to designing approval pathways for generic versions of recombinant protein-based therapeutics and monoclonal antibodies. Food Drug Law J. 2005;60(2):143-260.

2. Bhattacharyya L, Cecil T, Dabbah R, Roll D, Schuber S, Scheinin $\mathrm{EB}$, et al. The value of USP public standards for therapeutic products. Pharm Res. 2004;21(10):1725-31.

3. USP. USP 34-NF 29, General Notices. Rockville, MD: USP; 2011:34,6 .

4. SM Collaborative Group, Williams RL, Abernethy DR, Koch WF, Hauck WW, Cecil TL. Performance-based monographs. Pharmacopeial Forum. 2009;35(3):765-71.

5. Hauck WW, DeStefano AJ, Gecil TL, Abernethy DR, Koch WF, Williams RL. Acceptable, equivalent, or better: approaches for alternatives to official compendial procedures. Pharmacopeial Forum. 2009;35(3):772-8.

6. Koch WF, Hauck WW, de Mars SS, Williams RL. Measurement science for food and drug monographs: toward a global system. Pharm Res. 2010;27(7):1203-7.

7. Joint Committee for Guides in Metrology. International vocabulary of metrology - Basic and general concepts and associated terms (VIM). 3rd ed. Geneva: Joint Committee for Guides in Metrology; 2008.

8. ISO. Guide 32, calibration in analytical chemistry and use of certified reference materials. Geneva: ISO; 1997.

9. ISO. Guide 30: 1992/Amd 1:2008, terms and definitions used in connection with reference materials. 2nd ed. Geneva: ISO; 2008.

10. ISO. Guide 33, uses of certified reference materials. 2nd ed. Geneva: ISO; 2000. p. v.

11. ISO. International Standard 17025, general requirements for the competence of testing and calibration laboratories, 2nd ed. Geneva: ISO; 2005:5.4.5.2.

12. WHO. Technical Report 943 , WHO committee on specifications for pharmaceutical preparations, Annex 3, general guidelines for the establishment, maintenance, and distribution of chemical reference standards. Geneva: WHO; 2007. p. 62. 
13. WHO. Technical Report 932, WHO expert committee on biological standardization. Annex 2, recommendations for the preparation, characterization, and establishment of international and other biological reference standards. Geneva: WHO; 2005. p. 194-5.

14. WHO. International biological reference preparations. Geneva: WHO; 2011.

15. WHO. Technical Report 902, WHO expert committee on specifications for pharmaceutical preparations. Annex 3, good practices for National Pharmaceutical Control Laboratories. Geneva: WHO; 2002. p. 65.

16. FDA. Guidance for industry: analytical procedures and methods validation - chemistry, manufacturing, and controls documentation. Rockville: FDA; 2000.

17. FDA. Biotechnology inspection guide reference materials and training aids. Rockville: FDA; 2000.

18. FDA. Compliance program guidance manual 7356.002F: Active Pharmaceutical Ingredients (APIs). Rockville: FDA; 1998.

19. FDA, CDER. Human drug CGMP notes. 1997;5(2).

20. ICH. Q7: Good manufacturing practice guide for active pharmaceutical ingredients. Geneva: ICH; 2000.

21. USP. USP 34-NF 29, USP reference standards <11> Rockville: USP; 2011. p. 39-40.

22. European Department for the Quality of Medicine. European Pharmacopoeia, 5.12 Reference Standards. Strasbourg, France: EDQM; 2006:(suppl 5.6):4455-4458.

23. European Parliament. Directive $98 / 79 /$ EC of the European parliament and of the council of 27 October 1998 on in vitro diagnostic medical devices, Annex I. Brussels: EP; 1998.
24. World Health Organization and Food and Agriculture Organization of the United Nations. Codex Alimentarius, procedure manual. 19th ed. Geneva: World Health Organization; 2010.

25. International Conference on Harmonization. Q8(R2): Pharmaceutical development. Geneva: International Conference on Harmonization; 2009.

26. International Conference on Harmonization. Q9: Quality risk management. Geneva: International Conference on Harmonization; 2005.

27. International Conference on Harmonization. Q10 pharmaceutical quality system. Geneva: International Conference on Harmonization; 2008.

28. International Conference on Harmonization. Q11: Development and manufacture of drug substances. Geneva: International Conference on Harmonization; 2008.

29. Hauck WW, Koch W, Abernethy D, Williams RL. Making sense of trueness, precision, accuracy, and uncertainty. Pharmacopeial Forum. 2008;34(3):838-42.

30. Horowitz W. Nomenclature of interlaboratory analytical studies. Pure Appl Chem. 1994;66(9):1903-11.

31. Reference Standards Expert Committee Subcommittee on Certified Reference Materials, Manning RG, Lane S, Dressman S, Hauck WW, Williams RL. The application of uncertainty to USP's compendial reference standards program: certified reference materials. Pharmacopeial Forum. 2007;33:1300-6.

32. Williams RL, Abernethy DR, Koch WF, Hauck WW, Cecil TL. Performance-based monographs. Pharmacopeial Forum. 2009;35 (3):765-71. 